\title{
Obstetric Hemorrhage Volume and Severe Morbidity: A Retrospective Cohort Study
}

\author{
Michelle Wang ${ }^{1}$, Megan Alexander ${ }^{2}$, Diana Abbas $^{2}$, Akanksha Srivastava ${ }^{2}$, Ashley \\ Comfort $^{1}$, Ronald Iverson ${ }^{1}$, Howard Cabral ${ }^{3}$, and Christina Yarrington ${ }^{1}$ \\ ${ }^{1}$ Boston Medical Center \\ ${ }^{2}$ Boston University School of Medicine \\ ${ }^{3}$ Boston University
}

March 6, 2022

\begin{abstract}
OBJECTIVE: Our goal is to describe the association between total quantitative blood loss (QBL) and risk of obstetric hemorrhage-related morbidity (OBH-M) in order to assess utility of the current definition of obstetric hemorrhage (OBH). DESIGN: Retrospective cohort study. SETTING: Urban safety-net hospital in Boston, Massachusetts. POPULATION or SAMPLE: Deliveries at our institution over a two-year period. METHODS: We categorized deliveries into ten equally sized deciles based on QBL and compared the proportion with OBH-M in each. Among the two deciles with the highest proportions of OBH-M, we stratified deliveries into seven groups of ascending intervals of 250cc QBL. Finally, we compared the positive predictive value (PPV) of the standard definition of OBH (QBL [?] 1000cc) to a definition extrapolated from our stratified analysis. MAIN OUTCOME MEASURES: The primary outcome was proportion of deliveries within each QBL decile affected by OBH-M. The secondary outcome was PPV. RESULTS: We found a significant increase in OBH-M from decile 9 (895-1201cc QBL) to decile $10(1205-8325 \mathrm{cc}$ QBL) $(\mathrm{p}<0.001)$. In our stratified analysis, we found QBL of $1500 \mathrm{cc}$ to be an inflection point for an increased proportion of OBH-M. Our secondary analysis showed an increased PPV for OBH-M using QBL of 1500cc (20.5\%) compared to that of QBL 1000cc (9.8\%). CONCLUSIONS: Our findings suggest that a higher QBL threshold than the currently accepted definition of $\mathrm{OBH}$ is more predictive of OBH-M.
\end{abstract}

\section{Obstetric Hemorrhage Volume and Severe Morbidity: A Retrospective Cohort Study}

Short Title: Obstetric Hemorrhage Volume and Severe Morbidity

Michelle J. Wang, $\mathrm{MD}^{1,2}$

Megan Alexander, $\mathrm{MS}^{2}$

Diana Abbas, $\mathrm{BA}^{2}$

Akanksha Srivastava, $\mathrm{BS}^{2}$

Ashley Comfort, $\mathrm{MD}^{1,2}$

Ronald Iverson, $\mathrm{MD}^{1,2}$

Howard J. Cabral, $\mathrm{PhD}^{3}$

Christina Yarrington, $\mathrm{MD}^{1,2}$

${ }^{1}$ Boston Medical Center, Department of Obstetrics and Gynecology, Boston, MA.

${ }^{2}$ Boston University School of Medicine, Boston, MA. 
${ }^{3}$ Boston University School of Public Health, Department of Biostatistics, Boston, MA.

CONFLICTS OF INTEREST : The authors report no conflict of interest.

Acknowledgment: This work was supported in part by the Boston University Clinical and Translation Science Institute (NIH grant: 1UL1-TR001430).

\section{Corresponding author:}

Michelle J. Wang

michellejoyw@gmail.com

Boston Medical Center

Department of Obstetrics \& Gynecology

771 Albany Street, Dowling 4

Boston, MA 02118

Phone: 617-414-5175 | Fax: 617-414-2302

\section{ABSTRACT [Word Count: 226]}

OBJECTIVE: Our goal is to describe the association between total quantitative blood loss (QBL) and risk of obstetric hemorrhage-related morbidity (OBH-M) in order to assess utility of the current definition of obstetric hemorrhage $(\mathrm{OBH})$.

DESIGN: Retrospective cohort study.

SETTING: Urban safety-net hospital in Boston, Massachusetts.

POPULATION or SAMPLE: Deliveries at our institution over a two-year period.

METHODS: We categorized deliveries into ten equally sized deciles based on QBL and compared the proportion with OBH-M in each. Among the two deciles with the highest proportions of OBH-M, we stratified deliveries into seven groups of ascending intervals of 250cc QBL. Finally, we compared the positive predictive value (PPV) of the standard definition of $\mathrm{OBH}$ (QBL [?] 1000cc) to a definition extrapolated from our stratified analysis.

MAIN OUTCOME MEASURES: The primary outcome was proportion of deliveries within each QBL decile affected by OBH-M. The secondary outcome was PPV.

RESULTS: We found a significant increase in OBH-M from decile 9 (895-1201cc QBL) to decile 10 (1205$8325 \mathrm{cc}$ QBL) $(\mathrm{p}<0.001)$. In our stratified analysis, we found QBL of $1500 \mathrm{cc}$ to be an inflection point for an increased proportion of OBH-M. Our secondary analysis showed an increased PPV for OBH-M using QBL of $1500 \mathrm{cc}(20.5 \%)$ compared to that of QBL 1000cc (9.8\%).

CONCLUSIONS: Our findings suggest that a higher QBL threshold than the currently accepted definition of $\mathrm{OBH}$ is more predictive of OBH-M.

FUNDING: This work was supported in part by the Boston University Clinical and Translation Science Institute (NIH grant: 1UL1-TR001430).

KEYWORDS: obstetric hemorrhage, maternal morbidity, quantitative blood loss

TWEETABLE ABSTRACT (110 CHARACTERS) : The QBL associated with severe morbidity may be higher than the currently accepted definition of OBH.

\section{MANUSCRIPT}

INTRODUCTION 
The goal of defining a blood loss threshold that defines a significant obstetrical hemorrhage (OBH) is to find a sensitive and specific quantity of obstetrical bleeding that should trigger closer monitoring or interventions to prevent perinatal morbidity and mortality. ${ }^{1}$ Historical definitions of obstetric hemorrhage have primarily been based in expert-opinion rather than evidence-based thresholds of morbidity or mortality. ${ }^{2}$

The first proposed definition of postpartum hemorrhage traces back to a report published after an informal meeting of experts in 1989 at the World Health Organization, which defined PPH as "greater than 500mL blood loss from the genital tract after the delivery of a baby" but also acknowledged this as an "arbitrary figure" and "not always of great clinical significance." 2,3 In 2014, as part of the "reVITALize" initiative, the American College of Obstetricians and Gynecologists (ACOG) established a new definition of OBH as $1,000 \mathrm{cc}$ of cumulative blood loss regardless of route of delivery or specific vital sign changes associated with blood loss. ${ }^{4}$ This initiative focused on decreasing morbidity and mortality by standardizing the obstetrical definitions and approaches utilized by providers in the United States in the care of birthing people in labor. However, while a small cohort of historical data from Pritchard et al., which followed peripartum patients using 51 chromium labeled red cells, demonstrated mean losses to be around $500 \mathrm{~mL}$ in vaginal deliveries and around $1000 \mathrm{~mL}$ in cesarean deliveries, there is little contemporary data that demonstrates a specific association between obstetric blood loss of $1,000 \mathrm{cc}$ and clinically significant adverse perinatal outcomes. ${ }^{5}$

To address this "arbitrary" definition of OBH, we investigate the cumulative quantitative blood loss (QBL) associated with increased risk of severe obstetric hemorrhage-related morbidity (OBH-M). We also seek to compare the predictiveness for $\mathrm{OBH}-\mathrm{M}$ of the current definition of $\mathrm{OBH}$ to an alternate definition derived from our cohort.

\section{METHODS}

We performed a retrospective cohort study of all deliveries with a documented QBL from January 1, 2018, to January 1, 2020. Institutional review board approval was obtained from Boston Medical Center and Boston University School of Medicine.

At our institution, an interdisciplinary team implemented a postpartum hemorrhage bundle protocol in 2016 [Appendix S1]. It includes but is not limited to the following: unit preparation for obstetric hemorrhage, including simulations, hemorrhage risk assessment on admission [Appendix S2] and throughout the intrapartum course, quantitative blood loss (QBL) for all deliveries, and a staged response to the QBL.

Information was gathered from department birth logs that are abstracted daily from the electronic medical record for all deliveries. This includes information about maternal demographics, maternal medical and obstetric history, antenatal course, intrapartum course, anesthesia type if any, and QBL. Additional information was abstracted by trained chart abstractors into a standardized chart abstraction form using a secure database.

We created an OBH-M composite of all cases associated with any of the following: (i) transfusion of any blood products, (ii) intensive care unit admission, (iii) clinical, laboratory, or radiologic evidence of end-organ damage (iv) hysterectomy, (v) operative intervention in the immediate postpartum period, (vi) uterine artery embolization or (vii) maternal death. Individual deliveries were categorized into ten equally sized deciles based on the total QBL. We then compared the proportion of obstetric-hemorrhage related morbidity within each decile. Among the deciles with the highest proportions of OBH-M, we stratified deliveries into seven groups with ascending intervals of 250cc QBL to interrogate a threshold of QBL associated with an increase in association with a composite of obstetric hemorrhage-related morbidity (OBH-M). A significant point in change of risk for OBH-M was established by sequentially conducting the ranked analysis and stratified QBL-based analysis.

We then compared the standard definition of OBH ([?]1000cc) to the new definition extrapolated from our stratified analysis ([?]1500cc). These tests included positive predictive value (PPV), negative predictive value (NPV), sensitivity, specificity, and positive likelihood ratios (+LR). We utilized a McNemar test to compare sensitivity and specificity and calculated a post-test probability calculation based on the $+\mathrm{LR}$ values. We 
also calculated an area under the ROC curve (AUC) for each definition and compared the AUCs with the DeLong test. ${ }^{6}$ An alpha level of 0.05 was used to determine significance. All analyses were completed using STATA MP, version 17 or IBM SPSS Statistics $27.7,8$

\section{RESULTS}

There were 5243 deliveries in 2018-2019 with a documented quantitative blood loss. Our patient cohort characteristics are described in Table 1 (Table 1).

From our primary ranked analysis, we found that while there was a relatively stable low OBH-M frequency in deciles 1 through 8, there was a sharp increase from a mean frequency of OBH-M of three percent in decile 9 (895-1201cc QBL) to thirteen percent in decile 10 (1205-8325cc QBL) (Figure 1). The difference between decile 9 and 10 was found to be statistically significant $(\mathrm{p}<0.001)$.

As the range of significant inflection in $\mathrm{OBH}-\mathrm{M}$ appeared to be contained within deciles 9 and 10, we then compared OBH-M incidence of deliveries with a QBL greater than 750cc based on increments of 250cc QBL (Table 2). We found that deliveries with a QBL from $750-1000 \mathrm{cc}$ had a 2.4 percent rate of OBH-M with only a small increase to 3.7 percent for QBL ranging between 1001-1500cc (Table 2). There was a demonstrable inflection where the rate of OBH-M roughly doubled with every 250cc QBL interval: 7.2 percent OBH-M for QBL 1501-1750cc, 12.5 percent OBH-M for QBL 1751-2000cc, 25.0 percent OBH-M for QBL 2001-2250cc and 84 percent $\mathrm{OBH}-\mathrm{M}$ for $\mathrm{QBL}>2250 \mathrm{cc}$.

We then compared this to the accepted definition of 1000cc for hemorrhage to the threshold of 1500cc. The PPV for $1500 \mathrm{cc}$ was $20.5 \%$, compared to $9.8 \%$ for $1000 \mathrm{cc}$ (Table 3 ). With a sample prevalence of $2.0 \%$ for OBH-M within our cohort, there was an increased post-test probability of association of blood loss with OBH-M by $12 \%$ after increasing the threshold for hemorrhage to $1500 \mathrm{cc}$. While there was no statistical significance when the AUC of each definition was compared with the DeLong test $(\mathrm{p}=0.104)$ (Table 3), the "new" definition is clearly associated with an improved specificity at the cost of poorer sensitivity compared to the standard definition [Appendix S3].

\section{DICSCUSSION}

\section{Main Findings}

Our findings suggest that the QBL threshold that constitutes a clinically significant inflection point for increased association with OBH-M may be higher than suggested by currently accepted definitions of OBH.

\section{Strengths and Limitations}

A strength of our study was the use of equally sized decile groups for the analysis within our initial cohort analysis as opposed to comparing groups by various QBL cutoffs. This allowed us to find an initial inflection range of clinical significance in a less statistically arbitrary manner. Furthermore, as a hospital that was an early participant in the implementation of obstetric hemorrhage bundles, we are able to assess QBL results versus clinically important morbidities in a system with a standardized hemorrhage response. Finally, a strength of our study was the use of data measured using quantitative blood loss methods; most prior existing data informing clinical practices used blood loss quantified by visual estimation, which has been shown to significantly underestimate large volume blood loss by as much as $33-50 \%$ when compared to direct or quantitative measurement. ${ }^{9-12}$ Visually estimated blood loss is thus a poorly defined, inaccurate, and unreliable means of measuring morbidity associated with obstetrical bleeding.

Our study also has limitations. We did not create any adjusted models that would account for possible confounding factors that may impact either total blood loss or baseline risk for morbid outcomes. Future studies should consider stratifying results by factors such as delivery mode and underlying co-morbidities. We were also limited by only two years of data analyzed from a single institution with a population that may not have outcomes that are generalizable to all birthing people. However, as other institutions start to incorporate the use of postpartum hemorrhage bundle protocols that include the utilization of QBL, there is a great opportunity to replicate this data in the future with larger multi-site cohorts. 


\section{Interpretation}

What is the purpose of defining obstetric hemorrhage? The primary goals are: 1) to establish a sensitive blood loss threshold that triggers heightened clinical vigilance and 2) to establish aspecific indicator of severe perinatal morbidity and mortality. Perhaps a QBL of $1000 \mathrm{~mL}$ still functions well as a threshold that answers to the first goal; it is sensitive and inclusive, and triggers heightened clinical vigilance and the consideration of uterotonics or other interventions. At 1500 QBL, however, the incidence of OBH-M is up to 7\%, which is over three times the incidence of morbidity in the cohort at large and thus a more specific indicator for a severe morbidity event. Establishing a tiered threshold of and a corresponding staged response to obstetric hemorrhage may be a place to start in decreasing risk of and having accurate indicators for severe maternal morbidities. Using QBL of 1500cc may also be a helpful criterion to use in future research studies regarding $\mathrm{OBH}$ prevention and treatment.

\section{CONCLUSION}

Our study challenges the utility of the current accepted definition of obstetric hemorrhage and further demonstrates that perhaps a single definition of obstetric hemorrhage is not enough. ${ }^{1}$ Researchers and clinicians should utilize the ample morbidity and blood loss data accessible to state or region-based perinatal quality collaboratives and consortiums and international resources such as the World Health Organization Working Group on Maternal Mortality and Morbidity to continue to more accurately describe blood loss thresholds and confirm or update definitions to have the most optimum clinical utility.

\section{ACKNOWLEDGEMENTS}

NONE

\section{DISCLOSURE OF INTERESTS}

\section{NO CONFLICTS OF INTEREST}

\section{CONTRIBUTION OF AUTHORSHIP}

Michelle J. Wang contributed to the conceptualization of the research question, the gathering of data, the review of existing literature, the data analysis, and the writing and editing of the manuscript.

Megan Alexander contributed to data gathering, data analysis, and the editing of the manuscript.

Diana Abbas contributed to the data gathering, data analysis and the editing of the manuscript.

Akanksha Srivastava contributed to data gathering and editing of the manuscript.

Ashley Comfort contributed to data gathering, reviewing existing literature and editing of the manuscript.

Ronald Iverson contributed to research question conceptualization and manuscript editing.

Howard J. Cabral contributed to research question conceptualization, data analysis, manuscript editing.

Christina Yarrington contributed to research question conceptualization, to the review of existing literature and the editing of the manuscript.

\section{DETAILS OF ETHICS APPROVAL}

This study was approved by the Boston University / Boston Medical Center Institutional Review Board on IRB H-39914 on March 27, 2020.

\section{FUNDING}

Acknowledgment: This work was supported in part by the Boston University Clinical and Translation Science Institute (NIH grant: 1UL1-TR001430).

\section{REFERENCES}


1. Kerr RS and Weeks AD. Postpartum Haemorrhage: A Single Definition is No Longer Enough. BJOG. $2017 ; 124: 723-726$

2. World Health Organization. The Prevention and Management of Postpartum Haemorrhage: Report of a Technical Working Group, 3-6 July 1989. Geneva: WHO, 1990.

3. Dahlke JD, Mendez-Figueroa H, Maggio L, et al. Prevention and management of postpartum hemorrhage: a comparison of 4 national guidelines. Am J Obstet Gynecol. 2015; 213: 76.e1-10

4. Menard MK, Main EK, Currigan SM. Executive summary of the revitalize initiative: standardizing obstetric data definitions. Obstetrics \& Gynecology. 2014; 124(1):150-153.

5. Pritchard JA, Rowland RC. Blood volume changes in pregnancy and the puerperium. American Journal of Obstetrics and Gynecology. 1964; 88(3): 391-395.

6. DeLong, ER, DeLong, DM, Clarke-Pearson, DL. Comparing the Areas under Two or More Correlated Receiver Operating Characteristic Curves: A Nonparametric Approach. Biometrics. 1988; 44:837-845.

7. StataCorp. 2021. Stata Statistical Software: Release 17. College Station, TX: StataCorp LLC.

8. IBM Corp. Released 2020. IBM SPSS Statistics for Windows, Version 27.0. Armonk, NY: IBM Corp.

9. Bose P, Regan F, Paterson-Brown S. Improving the accuracy of estimated blood loss at obstetric haemorrhage using clinical reconstructions. BJOG. 2006; 113:919-924.

10. Patel A, Goudar SS, Geller SE, et al. Drape estimation vs. visual assessment for estimating postpartum hemorrhage. Int J Gynaecol Obstet. 2006; 93(3): 220-224.

11. Al Kadri HM, Al Anazi BK, Tamim HM. Visual estimation versus gravimetric measurement of postpartum blood loss: a prospective cohort study. Arch Gynecol Obstet. 2011; 283(6): 1207-1213.

12. Stafford I, Dildy G, Clark SL, Belfort MA. Visually estimated and calculated blood loss in vaginal and cesarean delivery. Am J Obstet Gynecol 2008; 199:519.e1-7.

\section{TABLES AND FIGURES CAPTION LIST}

\section{Figure 1. Frequency of Hemorrhage-Related Morbidity by QBL Deciles}

Figure Caption: When the proportion of deliveries associated with obstetric hemorrhage-morbidity in the $9^{\text {th }}$ decile $\left(2.34 \%\right.$ OBH-M) and $10^{\text {th }}$ decile $(12.76 \%$ OBH-M) were compared, the p-value was found to be $<0.0001$.

\section{Table 1. Study Population Demographics}

Table Caption :

Data are formatted n $(\%)$

*Unanswered for 1410 patients

+ Unknown for 17 patients

++ Unanswered for 38 patients

Table 2. Hemorrhage-Related Morbidity for Deliveries with a QBL > 750cc

Table Caption: N/A

Table 3. Table 3. Accuracy of Definitions of Hemorrhage Based on Blood Loss Threshold

Table Caption :

*P value for both sensitivity and specificity were found to be $<0.0001$ using the McNemar test. 
$+\mathrm{P}$ value comparing AUC values was found to be 0.1042 by DeLong test

\section{Supporting Information}

\section{Appendix S1. Boston Medical Center Obstetric Hemorrhage Bundle Components}

Appendix Caption:

Outline of Boston Medical Center Institutional Bundle structure modeled from the following resources:

California Maternal Quality Care Collaborative Hemorrhage Toolkit in National Partnership for Maternal Safety Hemorrhage Bundle Sections

Council on Patient Safety in Women's Health Care's Obstetric Hemorrhage Patient Safety Bundle

\section{Appendix S2. Boston Medical Center Obstetric Hemorrhage Risk Assessment Tool}

Appendix Caption: N/A

\section{Appendix S3. Area Under the Curve of Two Definitions of Obstetric Hemorrhage}

Appendix Caption: When the area under the ROC curve (AUCs) were compared using the DeLong test, the $\mathrm{P}$ value was found to be 0.1042 .

\section{Hosted file}

BJOG-TABLES.docx available at https://authorea.com/users/463769/articles/558810-obstetrichemorrhage-volume-and-severe-morbidity-a-retrospective-cohort-study

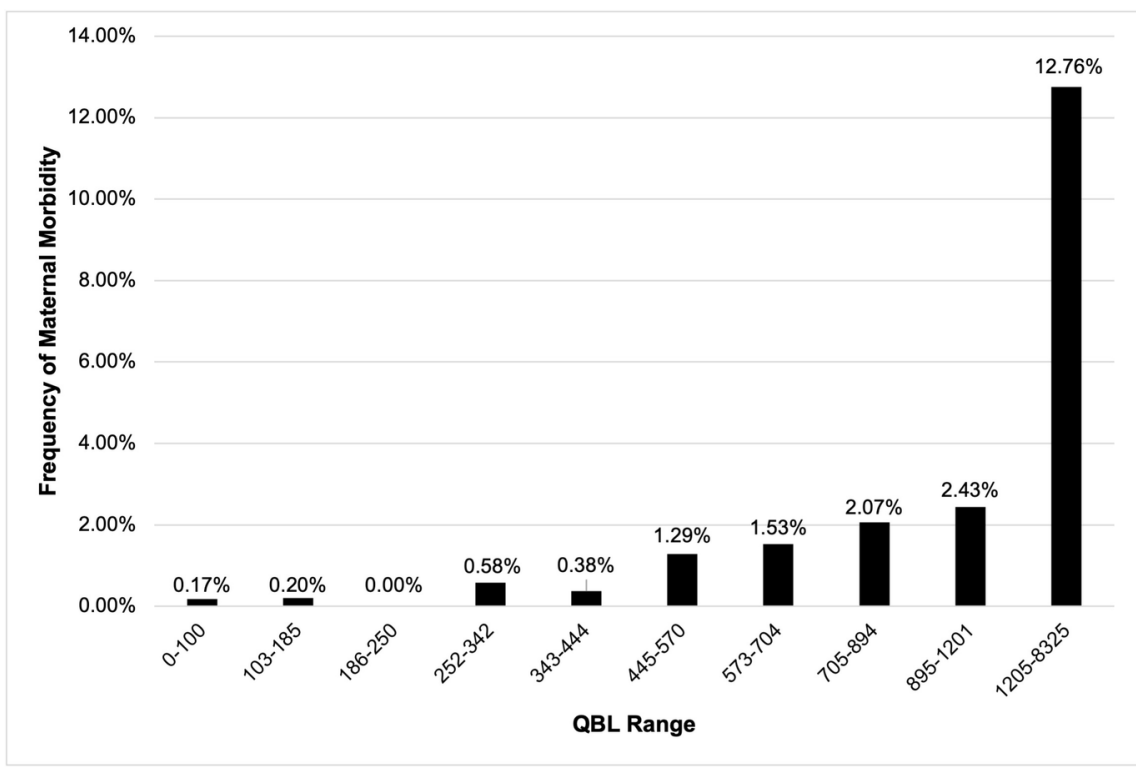

